\title{
Corrected Integral Shape Averaging Applied to Obstructive Sleep Apnea Detection from the Electrocardiogram
}

\author{
S. Boudaoud, ${ }^{1}$ H. Rix, ${ }^{1}$ O. Meste, ${ }^{1}$ C. Heneghan, ${ }^{2}$ and C. O'Brien $^{2}$ \\ ${ }^{1}$ Laboratoire d'Informatique, Signaux et Systèmes de Sophia Antipolis (I3S), UMR 6070 CNRS, 06903 Sophia Antipolis, France \\ ${ }^{2}$ School of Electrical, Electronic and Mechanical Engineering, University College Dublin, Belfield, Dublin 4, Ireland
}

Received 30 April 2006; Revised 31 October 2006; Accepted 1 November 2006

Recommended by William Allan Sandham

We present a technique called corrected integral shape averaging (CISA) for quantifying shape and shape differences in a set of signals. CISA can be used to account for signal differences which are purely due to affine time warping (jitter and dilation/compression), and hence provide access to intrinsic shape fluctuations. CISA can also be used to define a distance between shapes which has useful mathematical properties; a mean shape signal for a set of signals can be defined, which minimizes the sum of squared shape distances of the set from the mean. The CISA procedure also allows joint estimation of the affine time parameters. Numerical simulations are presented to support the algorithm for obtaining the CISA mean and parameters. Since CISA provides a well-defined shape distance, it can be used in shape clustering applications based on distance measures such as $k$-means. We present an application in which CISA shape clustering is applied to P-waves extracted from the electrocardiogram of subjects suffering from sleep apnea. The resulting shape clustering distinguishes ECG segments recorded during apnea from those recorded during normal breathing with a sensitivity of $81 \%$ and specificity of $84 \%$.

Copyright (c) 2007 S. Boudaoud et al. This is an open access article distributed under the Creative Commons Attribution License, which permits unrestricted use, distribution, and reproduction in any medium, provided the original work is properly cited.

\section{INTRODUCTION}

In the field of electrocardiogram (ECG) analysis, signal shape (or morphology) analysis is often used intuitively by clinicians for tasks such as beat typing and ischemia detection. However, there are relatively few well-defined analytical tools for automatically quantifying shape and shape differences, which can be used to capture the intuition of clinical practitioners, or to systematically uncover subtle but clinically significant changes in shape. One approach to signal shape quantification assumes a common underlying shape or template which is only subject to time warping $[1,2]$, and attempts to calculate both the time-warping function and a common template under a restrictive shape hypothesis. However, in practice, variations in shape from an underlying common shape are often the main objective of interest, and quantifying such shape variability is a goal of this work. In order to assess deviation from an underlying "template" shape, we first need to define the concept of an "averaged shape" reference signal. This signal should possess the necessary property of being a "shape gravity center" according to a specific shape distance (which we shall more precisely define in this paper). An implicit property of a well-defined shape distance will be to preserve shape equality under affine amplitude and time transformations of the reference signal. Given a shape distance and shape gravity center, it is then possible to clearly describe the shape statistics of a set of signals [3]. We note that a "shape center" defined by the simple classical mean, coupled with Euclidean distance will provide poor descriptors of shape variability, since both the mean and the distance measure are sensitive to time fluctuations $[2,4]$.

In order to provide a more robust technique for quantifying shape variability, we recently proposed a method called integral shape averaging (ISA) which has several useful properties $[2,4]$. For example, the ISA method applied to signals generated from a single shape reference signal, but with affine time and amplitude variations, will yield a shape reference signal which is equal to the original on an averaged time support. Furthermore, if the signals are different in shape, the ISA method will still provide a single shape reference signal, which can be used to form a similarity criterion between a single signal and the reference signal. This criterion is called the distribution functions method (DFM) criterion [5], and has been used to measure shape differences. Biomedical clustering applications have been carried out using a modified $k$-means clustering algorithm coupled with ISA signals and 
the DFM criterion $[6,7]$. While the results of this classification were in line with visual inspection, the theoretical basis of the method is somewhat uncertain due to the fact that the ISA signal does not possess the properties of a "shape gravity center". One of the reasons is because it is not invariant to affine time transforms. In addition, the DFM criterion is not a true distance measure.

A first attempt to remedy these shortcomings of the ISA method was proposed in [3] and applied in [8]. While providing practically useful clustering results, the proposed method did not fully correct the underlying theoretical problems. However, in this paper we provide a new approach, the corrected integral shape averaging (CISA), which addresses these issues. We specifically demonstrate that the CISA signal obtained by the method is a "shape gravity center" according to a CISA shape distance. We also provide an estimation procedure for the CISA model which obeys conditions for model identifiability. The proposed method permits modelling and estimation of both intrinsic shape fluctuation functions and affine time transforms. In Section 2, the analytical properties of the method are illustrated by numerical simulation.

As a specific example of how shape variability can be useful in a practical ECG classification problem, we use the CISA method for the task of recognizing obstructive sleep apnea (OSA) episodes based solely on analysis of the ECG. Obstructive sleep apnea is a common sleep disorder in which respiration is disordered during sleep due to partial or complete collapse of the upper airway. Its prevalence in the adult population is estimated at between 2-4\% [9], and it is linked with a number of unfavorable outcomes, such as increased risk of cardiovascular disease, hypertension, and excessive daytime sleepiness [10].

Sleep apnea is commonly defined as the cessation of breathing during sleep [11]. If breathing does not stop but the volume of air entering the lungs with each breath is significantly reduced, then the respiratory event is called a hypopnoea. Clinicians usually divide sleep apnea into three major categories: obstructive, central, and mixed apnea. Obstructive sleep apnea is characterized by intermittent pauses in breathing during sleep caused by the obstruction of the upper airway. The airway is blocked at the level of the tongue or soft palate, so that air cannot enter the lungs in spite of continued efforts to breathe. This is typically accompanied by a reduction in blood oxygen saturation and leads to wakening from sleep in order to breathe. Central sleep apnea (CSA) is a neurological condition which causes the loss of all respiratory effort during sleep and is also usually marked by decreases in blood oxygen saturation. With CSA, the airway is not necessarily obstructed. Mixed sleep apnea combines components of both CSA and OSA, where an initial failure in breathing efforts allows the upper airway to collapse. Currently, a definitive diagnosis of sleep apnea is made by counting the number of apnea and hypopnoea events over a given period of time (e.g., a night's sleep). Averaging these counts on a per-hour basis leads to commonly used standards such as the apnea/hypopnoea index (AHI) or the respiratory disturbance index (RDI). Polysomnog- raphy is used to measure these indices in clinical practice. The polysomnogram requires the recording of electroencephalogram, electrooculogram, and electromyogram to determine sleep stages, oronasal airflow, and chest-wall abdominal wall movements for respiratory effort, and oxygen saturation to monitor the effect of respiration and the electrocardiogram (ECG) for heart rate monitoring and arrhythmia screening. Typically, a full night's sleep is observed before a diagnosis is reached and in some subjects a second night's recording is required. Polysomnograms are expensive because they require overnight evaluation in sleep laboratories with dedicated systems and attending personnel. Due to the cost and relative scarcity of diagnostic sleep laboratories, it is estimated that sleep apnea is widely underdiagnosed [9]. Hence, techniques which provide a reliable diagnosis of sleep apnea with fewer and simpler measurements and without the need for a specialized sleep laboratory may be of benefit. Overnight unattended oximetry and airflow measurements have been investigated for this purpose, but there has also been an interest in using overnight ECG recordings from Holter monitors to screen for osbtructive sleep apnea [12]. The possibility of screening for OSA using ECG is based on (a) the known changes in RR intervals as the heart speeds up and slows down in response to apnea, and (b) QRS amplitude changes due to respiratory modulation of the ECG.

While results based solely on RR interval variability and the ECG-derived respiratory (EDR) signals are encouraging, we believe that sensitivity and specificity can be further improved by considering the fine structural changes in the ECG induced by apnea. Accordingly, we present a method to identify ECG segments associated with obstructive events by shape clustering of P-waves. Specifically, we extract the CISA average of $\mathrm{P}$-waves from apneic and nonapneic segments from ECG and demonstrate repeatable shape differences.

The main objective of this study is to confirm, in a more rigorous manner and using the proposed CISA formalism, the correlation between the P-wave shape and OSA occurrence.

\section{THE CISA METHOD}

\subsection{The model}

This paper provides a further theoretical development of the work first presented in [3]. The novel contribution is that rather than using the ISA signal as the shape reference signal, we now jointly estimate a shape reference signal, shape fluctuations, and affine parameters (scale and jitter) for a given model.

We first define the shape averaging problem, in the same manner as for our earlier development of the ISA method $[2,4]$. Assume that there are $N$ strictly positive signals $x_{i}(t)$, each being strictly positive on its support $\left[a_{i}, b_{i}\right]$. The case of nonpositive definite signals can also be practically dealt with by the addition of a suitable constant [4]. We assume that the signals are noise-free. The normalized integral (distribution 
function) can be defined as

$$
X_{i}(t)=\frac{\int_{a_{i}}^{t} x_{i}(u) d u}{\int_{a_{i}}^{b_{i}} x_{i}(u) d u}, \quad \forall i=1,2, \ldots, N,
$$

which is a monotonically increasing function of $t$. The distribution function $X_{i}$ can typically be linked to a shape reference signal $\tilde{S}$ through a time-warping function expressed as $\varphi$ or $\psi$. Specifically, we can write

$$
X_{i}=\tilde{S} \circ \varphi_{i}, \quad \tilde{S}=X_{i} \circ \psi_{i}, \quad \forall i=1,2, \ldots, N,
$$

where the notation $X_{i}=\tilde{S} \circ \varphi_{i}$ is shorthand for $X_{i}(t)=$ $\widetilde{S}(\varphi(t))$. The time-warping (which appears to be a monotonically increasing function) $\psi_{i}=\varphi_{i}^{-1}$ links the shape reference signal and the target signal $X_{i}$, and represents the fluctuations (in time and in shape or amplitude) [2]. A useful goal is to separate intrinsic shape variation from the variations caused by scale variation and jitter. To reach this objective, we propose a representation of $\varphi_{i}$ as

$$
\begin{gathered}
\varphi_{i}=v_{i} \circ A_{i}, \quad \psi_{i}=A_{i}^{-1} \circ \omega_{i}, \quad \forall i=1,2, \ldots, N, \\
v_{i}(t)=t+m_{i}(t), \quad v_{i}^{-1}(t)=\omega_{i}(t)=t+n_{i}(t), \quad \forall t,
\end{gathered}
$$

where $A_{i}(t)=\alpha_{i} t+\beta_{i}, \alpha_{i} \in \mathbb{R}^{+}, \beta_{i} \in \mathbb{R}$ is an affine function that accounts for variability in the time dimension (scale and jitter). The second element $v_{i}$ is a monotonically increasing nonlinear function that represents shape fluctuations on a constant time support. Its inverse function can be decomposed into the identity, and a function $n_{i}$ that represents nonlinear behavior. Both time and shape elements can be considered as a time-warping function linking two distribution functions. Therefore, we can rewrite (2) as follows :

$$
X_{i}=\tilde{S} \circ v_{i} \circ A_{i}, \quad \tilde{S}=X_{i} \circ A_{i}^{-1} \circ \omega_{i}, \quad \forall i=1,2, \ldots, N
$$

We define the proposed model relating a sample $X_{i}$ and the shape reference signal as the corrected integral shape averaging (CISA) model and the signal shape reference $\widetilde{S}$ as the CISA signal in $\mathbb{F}$. To ensure unique existence and parametrization of the CISA model shape reference, we impose the following conditions (see the appendix for details):

$$
\begin{aligned}
& \frac{1}{N} \sum_{i=1}^{N} \frac{1}{\alpha_{i}}=1, \quad \alpha_{i} \in \mathbb{R}^{+}, \quad \frac{1}{N} \sum_{i=1}^{N} \frac{\beta_{i}}{\alpha_{i}}=0, \quad \forall i=1, N, \\
& n_{i}\left(t_{\text {inf }}\right)=0, \quad n_{i}\left(t_{\text {sup }}\right)=0, \quad \frac{1}{N} \sum_{i=1}^{N} n_{i}(t)=0, \quad \forall t, \quad \forall i,
\end{aligned}
$$

where $t_{\text {inf }}$ and $t_{\text {sup }}$ are the limits of the CISA signal time support. The conditions on the function $n_{i}$ ensure the absence of compensatory effects between the shape fluctuation term and the affine term and also the shape averaging property of the CISA signal. In other words, the shape fluctuation term cannot change the signal time support when applied. The condition on the affine parameters permits the mean-time support recovery. The composition of the two elements of $\varphi_{i}$ can then be interpreted as a shape fluctuation warped with the affine time function $A_{i}$ applied on the distribution function $X_{i}$. This modeling is coherent with the reality where amplitude and phase variations are mixed in signal generation processes. Thanks to the integration operation, it is possible to regroup the two effects into one time function $\varphi_{i}$. To view the effect of the two components in the signal domain, we have to perform a time derivation (denoted by ') on $X_{i}$ to obtain

$$
X_{i}^{\prime}=\alpha_{i}\left(v_{i}^{\prime} \circ A_{i}\right)\left(\widetilde{S^{\prime}} \circ v_{i} \circ A_{i}\right), \quad \forall i=1,2, \ldots, N .
$$

Equation (6) shows how shape and time fluctuations of the original reference shape combine to give overall shape variation in a resulting signal. We note that the affine time transform acts on both the signal $x_{i}$ and its distribution function in the same manner. If we express (4) in the inverse domain $\mathbb{F}^{-1}$, we obtain:

$$
X_{i}^{-1}=\psi_{i} \circ \tilde{S}^{-1}=A_{i}^{-1} \circ \omega_{i} \circ \tilde{S}^{-1}, \quad \forall i=1,2, \ldots, N .
$$

Replacing in (7) $A_{i}^{-1}$ and $\omega_{i}$ with their expression in $y \in$ $[0,1]$, we obtain

$$
X_{i}^{-1}(y)=\frac{\left\{\tilde{S}^{-1}(y)+n_{i}\left\{\tilde{S}^{-1}(y)\right\}-\beta_{i}\right\}}{\alpha_{i}}, \quad \forall i=1, N
$$

We can rewrite this last equation in the form

$$
\tilde{S}^{-1}(y)=\alpha_{i} X_{i}^{-1}(y)+\beta_{i}-n_{i}\left\{\tilde{S}^{-1}(y)\right\}, \quad \forall i=1, N .
$$

In the following work, we will use (9) to model the unknown quantities. In fact, the time parameters, $\alpha_{i}$ and $\beta_{i}$, provide a linear regression between the reference shape signal and an arbitrary $X_{i}^{-1}$, with the additional nonparametric term $n_{i}\left\{\tilde{S}^{-1}(y)\right\}$ representing the shape fluctuation. In the next section, we present an estimation method that uses this equation to jointly model all the unknowns. Theoretical presentation of the CISA model dealt with continuous supported signals but in applications the mathematical expressions will be transferred to discrete domain, in the following sections, without any particular restrictions.

\subsection{Joint estimation of CISA model parameters and functions}

We use (9) for estimating the model parameters and functions. For this purpose, we rewrite it including an additional noise term $\varepsilon_{i}(y)$ of the form

$$
\begin{array}{r}
\mu(y)=\alpha_{i} z_{i}(y)+\beta_{i}+w_{i}(y)+\varepsilon_{i}(y), \\
\forall i=1,2, \ldots, N, y \in[0,1],
\end{array}
$$

where $\mu=\widetilde{S}^{-1}$ is defined as a CISA signal in $\mathbb{F}^{-1}, z_{i}=X_{i}^{-1}$, and $w_{i}=-n_{i} \circ \widetilde{S}^{-1}$. We assume that the noise sequence is a zero-mean i.i.d process with Gaussian distribution. We propose to estimate the model unknowns by using the "Procrustes" method adapted to the signal registration problem 
$[1,13,14]$. This consists of forcing a sample of signals to fit a signal reference while minimizing a specific cost function. The method is iterative and generally uses the conventional mean signal as a reference in the initialization step. Thereafter, the reference signal is updated until convergence. This procedure is adapted to our case where the warping operation is done by amplitude correction since it is performed in the inverse domain $\mathbb{F}^{-1}$. Indeed, the principal advantage of working with (10) rather than (4) is the fact that the model unknowns $\left(\alpha_{i}, \beta_{i}, w_{i}, \mu\right)$ appear linearly. Using (10), we propose to estimate the CISA signal and the other terms by minimizing the following cost function defined as the average integrated square error (AISE):

$$
\begin{aligned}
& \min _{\mu,\left(\alpha_{i}, \beta_{i}\right), w_{i}} \operatorname{AISE}_{N} \\
& =\min \left\{\frac{1}{N} \sum_{i=1}^{N} \int_{0}^{1}\left\{\mu(y)-\left[\alpha_{i} z_{i}(y)+\beta_{i}+w_{i}(y)\right]\right\}^{2} d y\right\},
\end{aligned}
$$

where the term $\alpha_{i} z_{i}(y)+\beta_{i}$ corresponds to the registered signals under an affine transform only and $w_{i}(y)$ is the shape difference term that remains to ensure the strict equality to the CISA signal. From an implementation point of view, we will often be dealing with discretely sampled sequences rather than functions, in which case, we can assume that the model functions are sampled uniformly on a linear grid defined in $[1, M]$ (corresponding to $[0,1]$ ). In such a case, we can rewrite the cost function as

$$
\operatorname{AISE}_{N, M}=\frac{1}{N} \sum_{i=1}^{N} \sum_{j=1}^{M}\left\{\mu(j)-\left[\alpha_{i} z_{i}(j)+\beta_{i}+w_{i}(j)\right]\right\}^{2} .
$$

In general, this is a complex nonlinear optimization problem, with a large number of free parameters and functions to optimize over. For example, in (12) there are a total of $M+2 N+M N$ free parameters. To reduce this high degree of freedom, we will impose several constraints on the model unknowns. We propose to minimize the proposed cost function iteratively following a multistage algorithm which will include estimation of the time parameters, shape fluctuation functions, and the CISA signal. For the sake of clarity, the iteration index is omitted. The algorithm steps are

Step 1 (initialization).

$$
-\hat{\mu}=\overline{z_{i}} \text { (initialization with the ISA signal). }
$$$$
-\widehat{w}_{i}(j)=0, i=1,2, \ldots, N, j=1,2, \ldots, M \text {. }
$$

Step 2 (time parameters estimation). The time parameters $\alpha_{i}$ and $\beta_{i}$ are estimated for each value of $i$ by least-square minimisation of a linear regression. The resulting expressions are

$$
\begin{aligned}
& \hat{\alpha}_{i}=\frac{N\left(\sum \hat{\mu} z_{i}-\sum \widehat{w}_{i} z_{i}\right)+\sum z_{i}\left(\sum \hat{w}_{i}-\sum \hat{\mu}\right)}{N \sum z_{i}^{2}-\left(\sum z_{i}\right)^{2}}, \\
& \hat{\beta}_{i}=\frac{\sum z_{i}^{2}\left(\sum \hat{\mu}-\sum \hat{w}_{i}\right)-\sum z_{i}\left(\sum \hat{w}_{i} z_{i}-\sum \hat{\mu} z_{i}\right)}{N \sum z_{i}^{2}-\left(\sum z_{i}\right)^{2}},
\end{aligned}
$$

where the sums are taken over $j=1$ to $M$ and $\hat{\mu}$ and $\widehat{w}_{i}$ are the estimated signal shape reference and shape fluctuation function in the preceding iteration, respectively. We apply the constraint on the affine functions $\left((1 / N) \sum A_{i}^{-1}(t)=t\right)$ by the following procedure:

$$
\widetilde{A}_{i}^{-1}=\widehat{A}_{i}^{-1}-\overline{\widehat{A}_{i}^{-1}}+\mathbf{I}
$$

where the function $\mathbf{I}$ is the identity function, $\overline{\hat{A}_{i}^{-1}}=$ $1 / N \sum_{i=1}^{N} \hat{A}_{i}^{-1}$, and $\hat{A}_{i}^{-1}(t)=\left(t-\hat{\beta}_{i}\right) / \hat{\alpha}_{i}$ is the estimated inverse affine function. Following this step, we can define the affine-registered versions $g_{i}$ of the functions $z_{i}$ with the expression $\hat{g}_{i}=\widetilde{A}_{i} \circ z_{i}$ computed by linear interpolation.

Step 3 (shape fluctuation functions estimation). In order to estimate the shape fluctuation functions $w_{i}$, we propose to use the following expression:

$$
\widetilde{w}_{i}=\hat{\mu}-\widehat{g}_{i} .
$$

To ensure uniqueness of the model, it is necessary to impose some restrictions on $\widetilde{w}_{i}$. The first one ensures that $\tilde{w}_{i}$ begins and finishes at zero. Practically, this is done by removing the baseline $\hat{u}_{i}$ estimated using the two points $\left[y_{1}, y_{M}\right]$. We summarize the operation with the following equation:

$$
\widetilde{\widetilde{w}}_{i}=\widetilde{w}_{i}-\hat{u}_{i} \circ z_{i}
$$

The second one is the equivalent condition in $\mathbb{F}^{-1}$ of the constraint on $n_{i}$ defined in (4) which forces the shape fluctuation functions to be averaged on the CISA signal

$$
\widehat{w}_{i}=\widetilde{\widetilde{w}}_{i}-\overline{\widetilde{w}}_{i}
$$

where the term $\overline{\widetilde{w}}_{i}$ is the mean of the estimated functions $\widetilde{\widetilde{w}}_{i}$.

Step 4 (CISA signal estimation). In order to estimate $\mu$, we rewrite (12) in the following matrix form:

$$
\operatorname{AISE}_{N, M}=\frac{1}{N} \sum_{i=1}^{N}\left[\mu-\widehat{g}_{i}-\widehat{w}_{i}\right]^{T}\left[\mu-\widehat{g}_{i}-\widehat{w}_{i}\right],
$$

where $\mu=\left[\mu_{1} \mu_{2} \cdots \mu_{M}\right]^{T}, \hat{g}_{i}=\left[\widehat{g}_{i, 1} \widehat{g}_{i, 2} \cdots \hat{g}_{i, M}\right]^{T}$ and $\widehat{w}_{i}=$ $\left[\widehat{w}_{i, 1} \hat{w}_{i, 2} \cdots \widehat{w}_{i, M}\right]^{T}$ for convenience. After partial differentiation with respect to $\mu$ and setting equal to zero, $\left(\partial \mathrm{AISE}_{N, M} \backslash\right.$ $\partial \mu=\mathbf{0}$ ), we obtain the following estimate for the value of $\mu$ which minimizes the cost function (it is a minimum by inspection):

$$
\widehat{\mu}=\frac{1}{N} \sum_{i=1}^{N} \widehat{g}_{i}+\frac{1}{N} \sum_{i=1}^{N} \widehat{w}_{i} .
$$

We know that the last term of the equation is equal to zero (17), since this is one of the imposed constraints for model uniqueness. Hence we obtain the equation

$$
\hat{\mu}=\frac{1}{N} \sum_{i=1}^{N} \hat{g}_{i}
$$


For each iteration, the estimator of $\mu$ is the mean of the estimated registered signals $\hat{g}_{i}$.

Step 5 (algorithm convergence testing). We compute the cost function defined in (18) for each iteration. If the function converges to a stable minimum (subject to some numerical assessment), we stop iterating, otherwise we return to Step 2.

Following convergence, we obtain the CISA signal expression in $\mathbb{R}^{+}$by

$$
\widehat{\widetilde{s}}=\left[\hat{\mu}^{-1}\right]^{\prime}
$$

where the $[\cdot]^{\prime}$ operator expresses differentiation with respect to time. For each $i$, we can also obtain the estimated shape fluctuation function $\hat{n}_{i}$ in the time domain using the following expression:

$$
\hat{n}_{i}=-\widehat{w}_{i} \circ \hat{\mu}^{-1} \text {. }
$$

We can also compute the registered signals in $\mathbb{R}^{+}$by inversion and differentiation of $\hat{g}_{i}$ :

$$
\check{x}_{i}=\left[\hat{g}_{i}^{-1}\right]^{\prime}
$$

where we note that the obtained signals are normalized in area.

\subsection{Simulation}

In order to demonstrate that the proposed algorithm obtains good estimates of the time and shape fluctuation and the CISA signal, we conduct numerical simulations in which all these quantities are known in generating the set of model signals. We simulated $N=10$ strictly positive signals that emulate ECG P-waves in shape, and are noiseless. The signals are defined in the interval $T \in[0,9]$ on $M=450$ points. We generate two families of shape fluctuation functions $m_{i}$ corresponding to positive and negative Gaussian functions respectively. The scale and jitter parameters follow a linear progression. The simulated data obey the CISA model presented in Section 3.1. In Figure 1, we provide the simulated signals, the CISA signal, affine functions $A_{i}$, and shape fluctuation functions $n_{i}$. The jitter variation is generated according to $\beta_{i}=0.1(i-(N-1) / 2)$ and the scale variation is given by $\alpha_{i}=0.03(i-(N-1) / 2)+1$.

We estimate the CISA model parameters and functions from the simulated signals by applying the procedure defined in Section 2.2 after the integration and inversion operations to obtain the $X_{i}^{-1}=z_{i}$. The convergence criterion is fixed to $\triangle \mathrm{AISE}=10^{-5}$. The $M$ values of $y$ are equally sampled in the interval $[0.005,0.995]$. To apply the constraint of (16), we use the two points $\left[y_{2}, y_{M-1}\right]$ corresponding, respectively, to the beginning and the end of the $X_{i}^{-1}=z_{i}$ signals. The algorithm converges after 8 iterations giving the results presented in Figure 2 . We observe good estimation of the CISA model with a final registration cost equal to $\mathrm{AISE}=3.22 \times 10^{-7}$. We also compute the RMS error

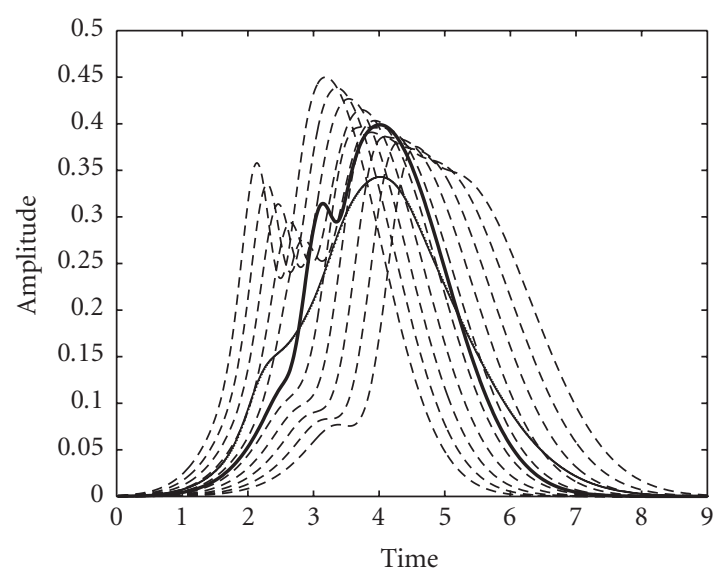

(a) Simulated signals (dashed curves), CISA signal (solid curve), and the mean (thick curve)

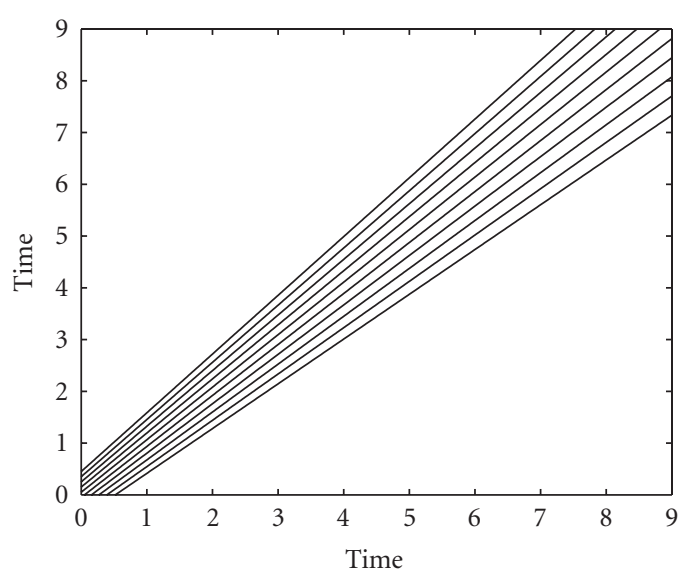

(b) The affine time functions $A_{i}$

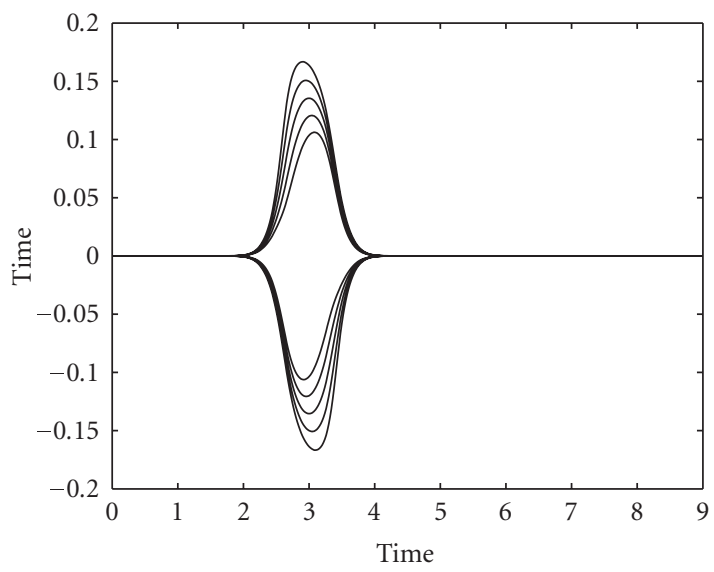

(c) The simulated shape fluctuation functions $n_{i}$

FIGURE 1: Simulation of signals using the CISA model.

between the CISA signal and its estimate and the ISA signal in $\mathbb{F}^{-1}$, respectively, as $e_{\mathrm{RMS}, \hat{\mu}}=3.2 \times 10^{-4}$ and $e_{\mathrm{RMS}, \mathrm{ISA}}=$ $5.77 \times 10^{-2}$. In addition, we calculated the normalized RMS 
error for the time parameter estimation computed as follows: $e_{\mathrm{RMS}, \hat{\alpha}_{i}}=\sqrt{(1 / N) \sum_{i=1}^{N}\left(\hat{\alpha}_{i}-\alpha_{i}\right)^{2} / \alpha_{i}^{2}}=8.59 \times 10^{-4}$ and $e_{\mathrm{RMS}, \hat{\beta}_{i}}=\sqrt{(1 / N) \sum_{i=1}^{N}\left(\hat{\beta}_{i}-\beta_{i}\right)^{2} / \beta_{i}^{2}}=2.27 \times 10^{-2}$. We can observe in Figure 2 the difference between the ISA and CISA signals. Although there are differences between the CISA and ISA methods, the ISA remains a good choice for the initialization procedure, and appears heuristically to place us close to the minimum of the cost function.

Moreover, the difference between signal reference shapes obtained using CISA and ISA methods can be explained theoretically. The expression for the ISA signal in $\mathbb{F}^{-1}$ is given by [2]

$$
\tilde{X}^{-1}=\frac{1}{N} \sum_{i=1}^{N} X_{i}^{-1}
$$

If we replace $X_{i}^{-1}$ by its expression in function of the CISA signal, we obtain

$$
\tilde{X}^{-1}=\frac{1}{N} \sum_{i=1}^{N}\left(A_{i}^{-1} \circ \omega_{i}\right) \circ \widetilde{S}^{-1}
$$

We replace also $A_{i}^{-1}$ and $\omega_{i}$ by their expression and apply the conditions $1 / N \sum_{i=1}^{N} \beta_{i} / \alpha_{i}=0$ and $1 / N \sum_{i=1}^{N} 1 / \alpha_{i}=1$ to finally obtain

$$
\tilde{X}^{-1}(y)=\tilde{S}^{-1}(y)+\frac{1}{N} \sum_{i=1}^{N}\left(\frac{n_{i}\left\{\tilde{S}^{-1}(y)\right\}}{\alpha_{i}}\right), \quad y \in[0,1] .
$$

From the equation, we explain the difference between ISA and CISA signals by the bias introduced by the last term of (26). In fact, the shape fluctuation functions are weighted by the scale parameters. Two important remarks can be made in relation to this point. First, if there is no shape fluctuation among signals, ISA and CISA signals are superimposed. Second, if there is only a jitter fluctuation, the two signals are also superimposed. Otherwise, the two signals are different and the CISA signal is the one that really averages the shapes on an average time support without additional time fluctuation contamination.

\subsection{Shape clustering using CISA}

Shape clustering provides a classification of signals based on their shape. In order to provide a useful classification, we need first to define the concept of shape equality. According to [15], the signals $x$ and $y$ are said to be "equal in shape" if and only if

$$
y(t)=a x\left(\frac{(t-\beta)}{\alpha}\right)+b
$$

Since the proposed method is based on signal area registration, we assume that $b=0$ for all signals or in other words, the offset term is removed before CISA calculation. Furthermore, to describe the shape statistics of a sample of signals while respecting the shape equality condition, one needs to define [3]:

(i) a distance $d$ between signals which is in fact a distance between the signal shapes. A natural consequence of this is that if we take two signals $x$ and $y$, then $d(x, y)=$ $0 \Leftrightarrow x$ and $y$ are "the same shape." Thus, this shape distance is invariant to affine transforms,

(ii) a sample mean that is intrinsically invariant in shape to the affine transforms described in (27), and hence a mean which provides a realistic average shape signal [3]. In addition, this mean shape signal must have the property of being a "shape gravity center" with respect to the shape distance $d$. We can easily demonstrate that the CISA signal coupled with the CISA distance has such properties. In fact, if we define the CISA distance between two signals $x_{1}$ and $x_{2}$ that belong to a sample of $N$ signals as

$$
d_{\mathrm{CISA}}\left(x_{1}, x_{2}\right)=\sqrt{\int_{0}^{1}\left(A_{1}\left\{X_{1}^{-1}(y)\right\}-A_{2}\left\{X_{2}^{-1}(y)\right\}\right)^{2} d y}
$$

then the CISA distance can be interpreted as the $L^{2}[0,1]$ norm in $\mathbb{F}^{-1}$ between the two registered and area-normalized signals. It can also be expressed as

$$
d_{\mathrm{CISA}}\left(x_{1}, x_{2}\right)=\sqrt{\int_{0}^{1}\left(n_{1}\left\{\tilde{S}^{-1}(y)\right\}-n_{2}\left\{\tilde{S}^{-1}(y)\right\}\right)^{2} d y} .
$$

In this last expression, the CISA distance is expressed as a function of the shape fluctuation referred to the CISA signal. This quantity does represent a true shape distance, as demonstrated by the following. Since the CISA signal is the mean of the registered signals $A_{i} \circ X_{i}^{-1}$ in $\mathbb{F}^{-1}$, we can write

$$
\tilde{S}^{-1}=\underset{u \in \mathbb{R}}{\arg \min } \sum_{i=1}^{N} d^{2}\left(u, A_{i} \circ X_{i}^{-1}\right)=\frac{1}{N} \sum_{i=1}^{N} A_{i} \circ X_{i}^{-1} .
$$

This last equation expresses the shape variance minimization property of the CISA signal. Indeed, the CISA signal is a "shape gravity center." As shown above, both the CISA signal and distance by definition are invariant to affine transforms.

To perform shape clustering, we can use the well-known $k$-means approach [16]. The method consists of separating signals into classes (clusters) that maximize interclass variance and minimize intraclass variance. It can alternatively be expressed as a global minimization problem in which the quantity to minimize is the sum of distances to the nearest neighbor. Classically, the method employs the conventional mean and the $L^{2}$ distance in $\mathbb{R}$ as class center and clustering 


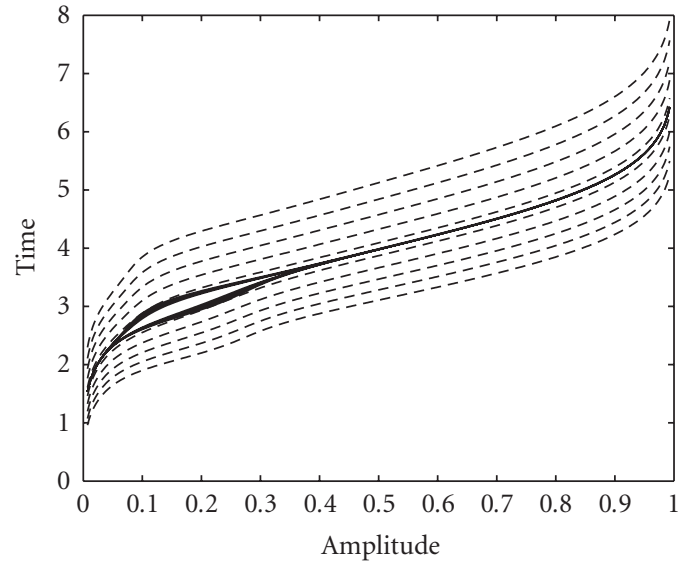

(a) The $X_{i}^{-1}=z_{i}$ signals (dashed line) and the registered $\hat{g}_{i}$ ones in $\mathbb{F}^{-1}$ (solid line)

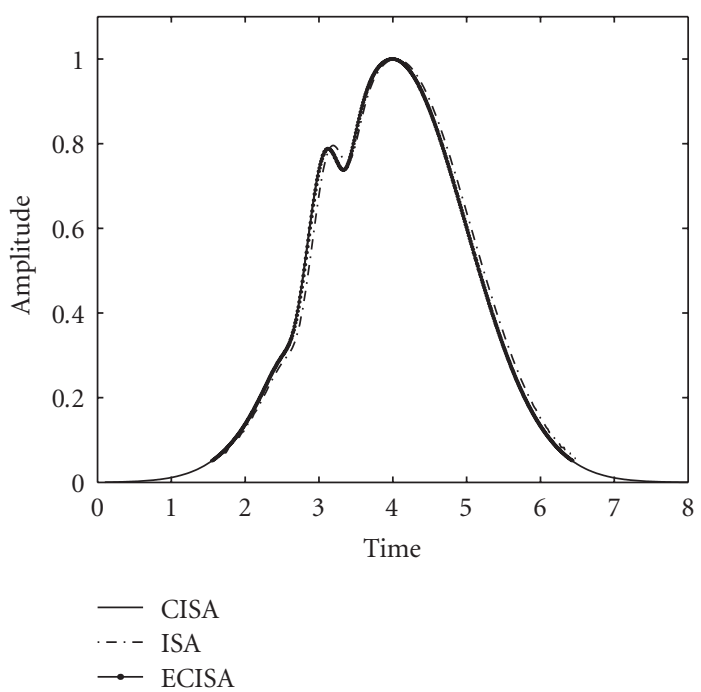

(c) The ISA, the theoretical CISA, and the estimated CISA signals
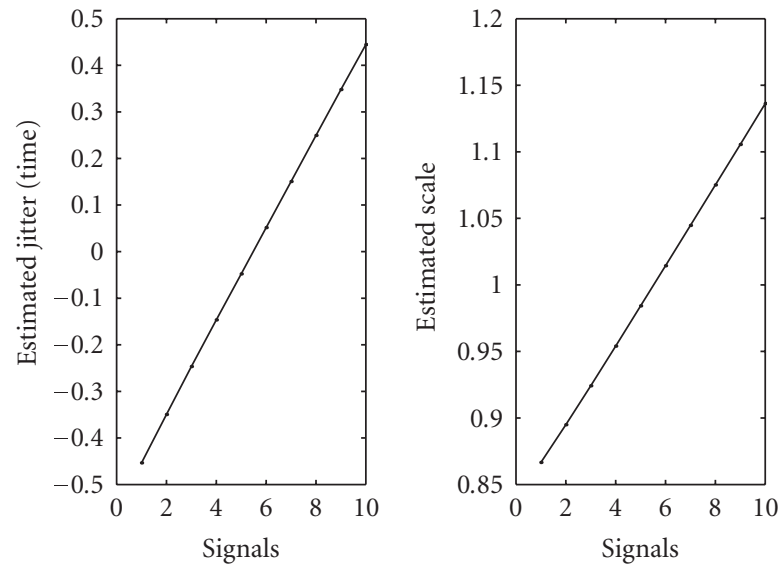

(b) The estimated jitter and scale parameters

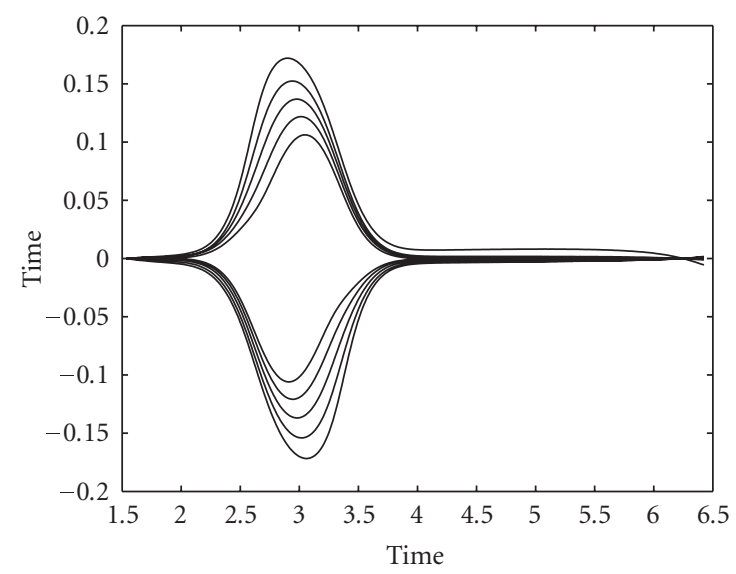

(d) The estimated shape fluctuation functions $\hat{n}_{i}$

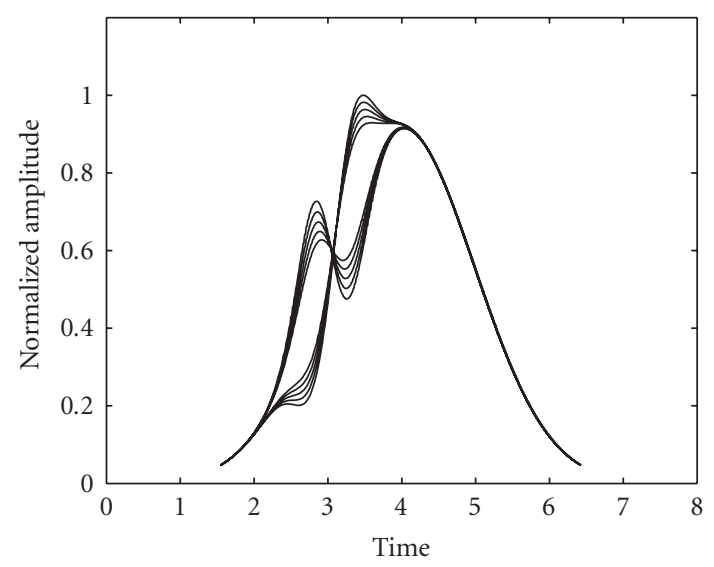

(e) The registered $\check{x}_{i}$ signals

Figure 2: Estimation of the CISA model from the simulated data. 


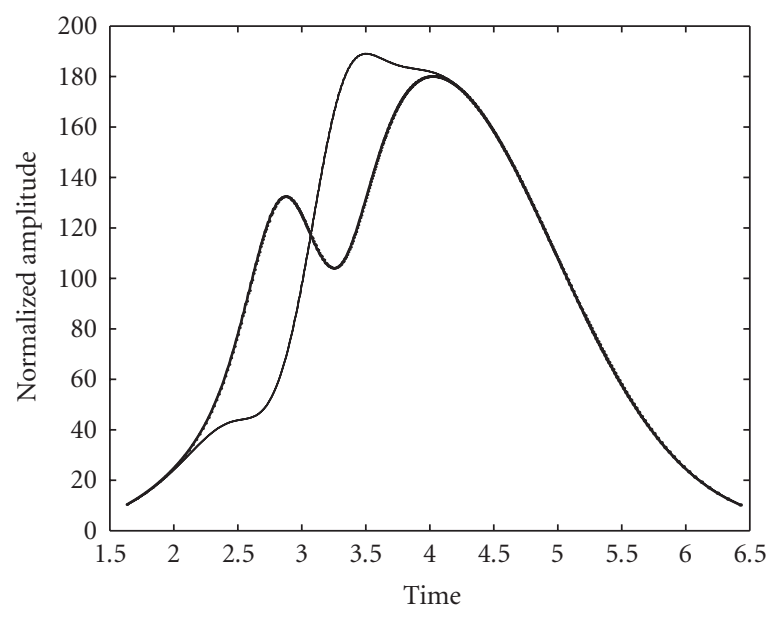

Figure 3: The final CISA class centers from the simulated data.

distance, respectively. In the presence of time fluctuations, these tools give bad clustering performances. To deal with the time fluctuations, we propose to use the $k$-means algorithm to cluster signal shapes in $\mathbb{F}^{-1}$, by using the CISA signal and distance as class center and distance, respectively. This has already been done for a biomedical application using the ISA approach $[6,7]$ using a similarity criterion rather than a distance. However, as mentioned previously, the ISA center does not necessarily possess a gravity center property.

To illustrate the utility of the CISA method, we first propose to apply it for clustering the signals of the preceding simulation example into two classes. Firstly, all the signals are registered to eliminate affine fluctuation. Then, the $k$ means algorithm is launched with random class center initialization chosen from among the $\check{x}_{i}$ signals. The CISA signal is then computed until convergence for each class and a discrete version of CISA distance is used. The procedure is repeated $L$ times. The final clustering solution is selected based on class separation criterion maximization and solution redundancy. The separation criterion represents the ratio between the final interclass center distance and the sum of both intraclass distance standard deviations. A ratio greater than 1 indicates good class separation. For our example, the 10 signals are well classified and the final class centers can be seen in Figure 3. The clustering criterion obtained is equal to $R=5.51$, indicating a high shape separation between the two classes. This validates that the CISA methodology can be used to provide accurate classification of signals based solely on shape differences. Indeed, the use of this parameter respects the unsupervised nature of the classification (no use of a priori information). Other criteria should be designed to measure classification performances of a particular application. Practically, affiliation of the obtained classes to the healthy and pathological ones should be done using some a priori information or specific characteristics of the class (shape dispersion). In our case study, we will assume that the classification done by the expert is perfect. So, the class affiliation will be done according to signals which lie mostly within their correct class.

\section{OBSTRUCTIVE SLEEP APNEA DETECTION}

As discussed in the introduction, obstructive sleep apnea (OSA) is a common sleep disorder with many physiological consequences, such as increased risk of cardiovascular disease, hypertension, and daytime sleepiness. Previously techniques have been developed to recognize periods of sleep apnea from the ECG by using RR interval variability, and an ECG-derived respiratory signal. However, no previous approaches have considered morphological changes of the ECG due to OSA. Such changes, especially for the P-wave or T-wave, could have an underlying physiological plausibility as ischemia due to oxygen desaturation could alter the electrical activation of the atria and ventricles $[17,18]$. In a recent study, we showed a strong correlation between Pwave shape changes and the occurrence of OSA events. However, this initial approach used an improvement of the ISA method (with consequent incomplete theoretical foundation as a shape clustering technique) and presented analysis on a small subset of signals. In this current work, we use the CISA methodology on a larger set of signals to provide a robust classification of ECG segments.

\subsection{P-wave shape clustering}

In this section, we discuss results on the use of the CISA signal and distance coupled with a $k$-means algorithm for developing an unsupervised shape classifier. The method is applied to perform a clustering [8] on P-wave shapes extracted from $163 \mathrm{ECG}$ segments sampled at $128 \mathrm{~Hz}$. These segments are each 2 minutes in length and were acquired from 7 subjects who suffer from OSA. For these segments, 95 are considered as normal and 68 are centered on a discrete episode of OSA of 10 second duration or longer. The ECG segments were extracted from complete polysomnogram recordings carried out at St. Vincent's University Hospital, Dublin, using the Jaeger-Toennies polysomnogram system.

The epoch labeling was obtained from the polysomnogram analysis by an expert. For each segment, the P-waves were segmented according to the QRS complex, baseline corrected after upsampling (by a factor of five) and spline filtering. The artifacts were also removed by amplitude threshold selection as follows: for each segment, a shape homogeneous $\mathrm{P}$-wave set was detected by shape analysis. In fact, the CISA procedure was performed for one iteration for each segment. For this purpose, discrete versions of both the CISA signal and distance expressions were used. After that, signals within one standard deviation of CISA distance from the CISA mean were selected. These signals were averaged to provide us with the overall segment shape prototype. This procedure avoids shape contamination of the segment representative signal by noisy signals (e.g., the P-wave corresponding to a premature atrial contraction or normal $\mathrm{P}$-waves inside an apneic segment). For each subject, the segment prototypes were then registered following the CISA procedure after adequate windowing procedure. The convergence criterion was fixed to $\triangle \mathrm{AISE}=10^{-5}$ and the inverse distribution interval was set to $y=[0.02,0.96]$. Then, a two-class (normal, apnea) clustering was done on the segment prototypes for 
TABLE 1: CISA shape clustering results.

\begin{tabular}{lccccc}
\hline Subject & No. of normal segments & No. of apnea segments & Sens. (\%) & Spec. (\%) & R \\
\hline 1 & 15 & 11 & 91 & 93 & 1.95 \\
2 & 10 & 8 & 87 & 80 & 1.94 \\
3 & 15 & 10 & 70 & 73 & 0.74 \\
4 & 10 & 10 & 60 & 100 & 2.83 \\
5 & 15 & 10 & 90 & 80 & 1.34 \\
6 & 15 & 10 & 80 & 86 & 1.35 \\
7 & 15 & 9 & 88 & 73 & 1.31 \\
\hline Average & - & - & 80.9 & 83.6 & - \\
\hline
\end{tabular}

$L=15$ trials following the procedure described in Section 2.3 for each subjects So ideally for a single subject represented (e.g.) with ten "normal" segments and twelve "apnea" segments we hope to achieve a clustering into two classes which have 10 and 12 members, respectively, each belonging correctly to the classes "normal" and "apnea." For the 7 subjects studied in this paper, the results are shown in Table 1. For each subject, the number of normal and apneic segments and the class separation criterion $\mathrm{R}$ value are indicated. These segment numbers correspond to the classification done by the expert and assumed to be perfect. In Figure 4, we show the original and registered P-wave prototypes using the estimated affine parameters for the recordings from Subject 2. We can also observe the final CISA class centers obtained by the approach. We can observe a significant shape difference between the two signals. For most subjects, the class separation is good according to the $\mathrm{R}$ value. As can be observed, the proposed method reached an overall sensitivity of $80.9 \%$ and specificity of $83.6 \%$ and maximum sensitivity of $91 \%$ and maximum specificity of $93 \%$. This result confirms the earlier results obtained on a smaller database in [8], the existence of a strong correlation between $\mathrm{P}$-wave shape variation and the occurrence of OSA.

\subsection{Relation between time variations of the P-wave and apnea}

In addition to shape fluctuation estimation, the CISA method also simultaneously permits an estimation of the affine time parameters (scale and jitter) used in the realignment procedure (see Section 2.2). It is plausible that changes in these parameters can be linked to OSA occurrence and also be used to recognize apnea episodes. Both scale and jitter were estimated from the segment prototypes of each subject following the estimation procedure described in Section 2.2. We then tried to find some correlation (by true classification) between the variation of these parameters and the labeling of the corresponding segments (normal or apneic) for each subject done by the expert. For two subjects, the scale parameter variation dominated and showed a clear increase in the P-wave duration during OSA. A scale threshold $\alpha_{\text {thr }}=1$ was used which maximizes the classification performance (based on maximizing of the sum of specificity and sensitivity) for the two classes (normal and apnea) of the concerned segment prototypes based solely on the scale estimated parame- ter. This value corresponds to the reference scale of the CISA signal. Indeed, scale and jitter parameters are relative values referring to the CISA signal. The classification was based on a simple threshold decision rule. If the scale parameter $\alpha_{i}$ is greater than one (signal dilation), this means that the corresponding segment is apneic; otherwise it is classified as normal. The results for this analysis are shown in Table 2 . In addition, for two other subjects, the effects of apnea were to provide combined jitter and scale variation. For the classification task, therefore, we used a parameter that mixes both scale and jitter $\gamma_{i}=\beta_{i} / \alpha_{i}$. For these two subjects, we tried to find a threshold $\gamma_{\text {thr }}$ that maximizes the classification performance. For a subject, if the parameter $\gamma_{i}$ is greater than $\gamma_{\mathrm{thr}}$, the signal is considered as apneic, otherwise it is normal. The results obtained are shown on Table 3 . In the three remaining subjects, time parameters variation was not significantly correlated to OSA occurrence. We conclude, therefore, that apnea episodes can affect both the time duration and timing of the P-wave (PR interval) but in a subject-specific fashion.

\section{CONCLUSIONS}

In this paper, we have presented a novel method, corrected integral shape averaging (CISA), for signal realignment and shape fluctuation estimation. The method is based on a signal model that includes both shape and time support variation of the signal distribution functions. The CISA approach provides us with a new mean shape signal, the CISA signal, that possesses interesting mathematical properties for signal shape characterization. In fact, coupled to the CISA distance, the CISA signal minimizes shape fluctuation variance over a set of signals to be averaged. In addition, it is not contaminated by affine time fluctuations as for a related method using integral shape averaging. These useful properties make the CISA signal a good candidate for shape clustering applications. The CISA model contains a number of unknown parameters relating to time and shape variation, and we have described an iterative estimation procedure for obtaining the values of these parameters. The performance of the CISA procedure is illustrated using a controlled numerical simulation, and it can be shown that it clearly classified synthetic signals into their correct classes despite the presence of time fluctuations. The method was then applied to evaluate the benefits of using P-wave shape to recognize obstructive sleep apnea in ECG recordings. Using the $k$-means algorithm, the 


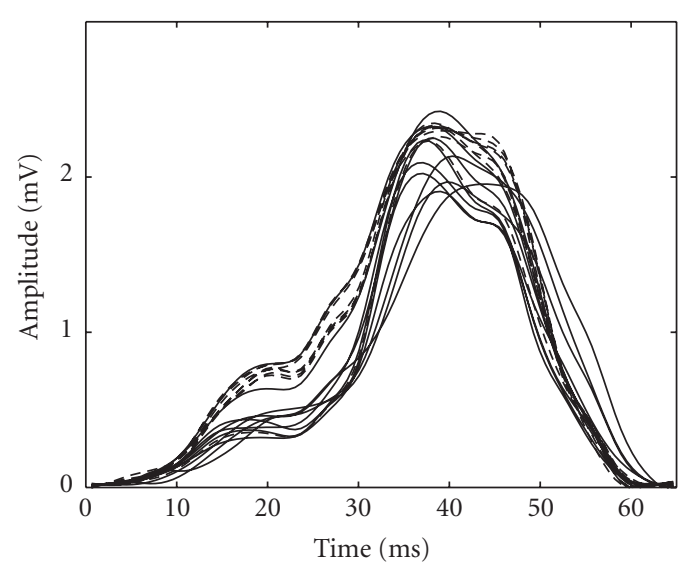

(a) The normal (-) and the apneic P-waves (- -) (according to expert labeling) before realignment

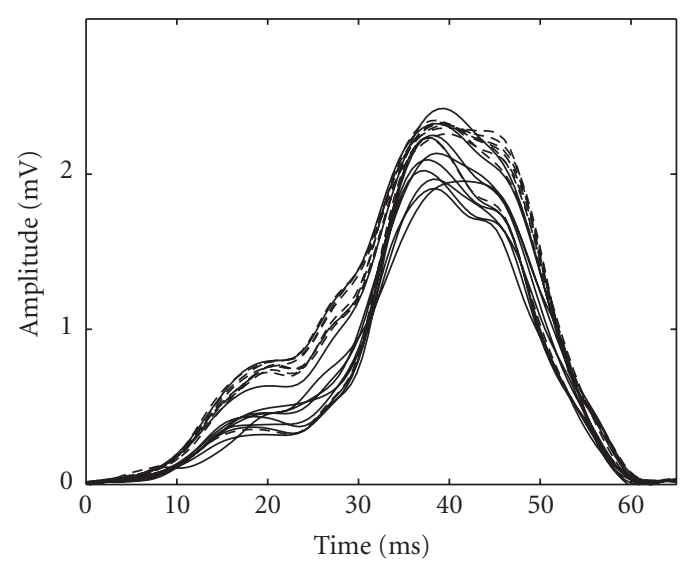

(b) The normal (-) and the apneic P-waves (--) after realignment

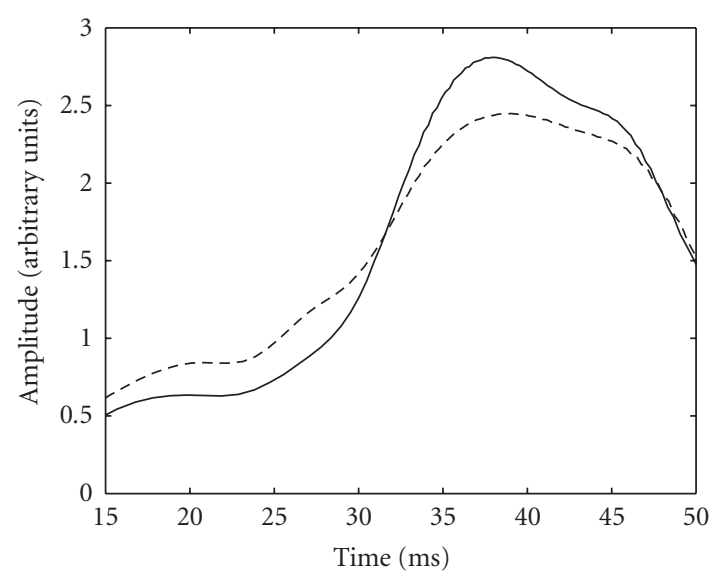

(c) The final CISA class centers (normal -, apneic--)

Figure 4: Results of CISA averaging technique applied to the P-wave segments measured in Subject 2.

method performed a clustering operation on a database of 163 signals. The classification results confirmed, in a more rigorous fashion, the important correlation linking P-wave shape and OSA episodes occurrence described in a previous work. A true classification procedure (based on the knowledge of the perfect classification) using the time parameters (scale and jitter) estimated by the CISA method was also proposed and applied on some subjects. The obtained results showed also a time alteration of the P-wave induced by OSA occurrence in a patient-specific manner.

A limitation of the study was the relatively low sampling rate for ECG, and we expect that future studies using ECG measurements with higher sampling rate would improve the shape analysis performances. Since the objective of the paper was the rigorous confirmation of the correlation between shape changes and apnea occurrence, the following step would be the design of an apnea "detector" using shape information. This device should use the P-wave shape variation also combined with the existing RR interval variability and EDR techniques to improve overall classification performance. However, the proposed CISA approach could also be used to analyze other bioelectrical signals such as other ECG components or brain-evoked potentials.

In conclusion, the CISA method and its application highlight the potential of signal shape analysis by time-warping estimation for signal description. In fact, both minimizing the signal parametrization and functional modeling permit a direct access to signal shape information and implicitly to generation process properties. In biomedical applications, dealing with shape and time warping may assist in providing plausible physiological models for signal generation.

\section{APPENDIX}

\section{CISA MODEL IDENTIFIABILITY}

In this section, we prove the identifiability of the proposed CISA model. For this purpose, we suppose that there exists two different solutions $\mu$ and $\mu^{\prime}$, that is, $z_{i}$ signals are linked to $\mu$ and $\mu^{\prime}$, respectively, by the following CISA model equations without noise in $\mathbb{F}^{-1}$ :

$$
\begin{aligned}
\mu(y)=\alpha_{i} z_{i}(y)+\beta_{i}+w_{i}(y), & \forall i=1: N, y \in[0,1], \\
\mu^{\prime}(y) & =\alpha_{i}^{\prime} z_{i}(y)+\beta_{i}^{\prime}+w_{i}^{\prime}(y), \quad \forall i=1: N, y \in[0,1] .
\end{aligned}
$$

If we replace $z_{i}$ expression from the first equation in the second one

$$
\mu^{\prime}(y)=\frac{\alpha_{i}^{\prime}}{\alpha_{i}} \mu(y)+\left[\beta_{i}^{\prime}-\frac{\beta_{i} \alpha_{i}^{\prime}}{\alpha_{i}}\right]+\left[w_{i}^{\prime}(y)-\frac{\alpha_{i}^{\prime}}{\alpha_{i}} w_{i}(y)\right] .
$$

We rewrite the equation to the form

$$
\mu^{\prime}(y)=A_{i} \mu(y)+B_{i}+g_{i}(y), \quad \forall i=1: N .
$$

The first term of the equation being independent of $i$, we can write for $i=k$ and $i=l$

$$
\begin{aligned}
& \mu^{\prime}(y)=A_{k} \mu(y)+B_{k}+g_{k}(y), \quad y \in[0,1] \\
& \mu^{\prime}(y)=A_{l} \mu(y)+B_{l}+g_{l}(y), \quad y \in[0,1] .
\end{aligned}
$$


TABLE 2: Classification based on scale parameter.

\begin{tabular}{lccccc}
\hline Subject & No. of normal segments & No. of apnea segments & Sens. (\%) & Spec. (\%) & $\alpha_{\text {thr }}$ \\
\hline 1 & 15 & 11 & 82 & 73 & 1 \\
2 & 10 & 8 & 87 & 80 & 1 \\
\hline Average & - & - & 84.5 & 76.5 & - \\
\hline
\end{tabular}

TABle 3: Classification based on both jitter and scale parameters.

\begin{tabular}{lccccc}
\hline Subject & No. of normal segments & No. of apnea segments & Sens. $(\%)$ & Spec. $(\%)$ & $\gamma_{\text {thr }}(\mathrm{ms})$ \\
\hline 4 & 10 & 10 & 80 & 80 & 0 \\
7 & 15 & 9 & 88 & 73 & 0.8 \\
\hline Average & - & - & 84 & 76.5 & - \\
\hline
\end{tabular}

We substract the second equation from the first

$$
\begin{aligned}
\left(A_{k}-A_{l}\right) \mu(y)+\left(B_{k}-B_{l}\right), & \\
+ & +\left(g_{k}(y)-g_{l}(y)\right)=0, \quad \forall y \in[0,1] .
\end{aligned}
$$

Then we apply the conditions $g_{i}(0)=0$ and $g_{i}(1)=0$ (from conditions on $w_{i}$ and $\left.w_{i}^{\prime}\right)$ for the two values $y=0$ and $y=1$ where $\mu(0)=t_{\text {inf }}$ and $\mu(1)=t_{\text {sup }}$ and obtain

$$
\begin{gathered}
\left(A_{k}-A_{l}\right) t_{\text {inf }}+\left(B_{k}-B_{l}\right)=0 \\
\left(A_{k}-A_{l}\right) t_{\text {sup }}+\left(B_{k}-B_{l}\right)=0 .
\end{gathered}
$$

From these two equations, we can deduce that $A_{k}=A_{l}=A$ and $B_{k}=B_{l}=B$ since $t_{\text {inf }} \neq t_{\text {sup }}$. In addition, we can add after replacing these last parameters in (A.5) that $g_{k}=g_{l}=g$ for all $y$. Finally, we rewrite (A.3) in the form

$$
\mu^{\prime}(y)=A \mu(y)+B+g(y)
$$

where $A=\alpha_{i}^{\prime} / \alpha_{i}, B=\beta_{i}^{\prime}-\beta_{i} \alpha_{i}^{\prime} / \alpha_{i}$, and $g(y)=w_{i}^{\prime}(y)-$ $\left(\alpha_{i}^{\prime} / \alpha_{i}\right) w_{i}(y)$ are quantities independent of indexed $i$.

Computing the mean of $A / \alpha_{i}^{\prime}$ and applying the conditions on the scale parameters in (5) give $A=\left(1 / N \sum_{i=1}^{N} 1 / \alpha_{i}\right) /$ $\left(1 / N \sum_{i=1}^{N} 1 / \alpha_{i}^{\prime}\right)=1$. This means that $\alpha_{i}=\alpha_{i}^{\prime}$ for all $i$.

Computing the mean of $B / \alpha_{i}^{\prime}$ and applying the conditions on both scale and jitter parameters in (5) give $B / N \sum_{i=1}^{N} 1 / \alpha_{i}^{\prime}=\left[1 / N \sum_{i=1}^{N} \beta_{i}^{\prime} / \alpha_{i}^{\prime}-1 / N \sum_{i=1}^{N} \beta_{i} / \alpha_{i}\right]=0$. Since $A=1$ and $B=0$, we obtain $\beta_{i}=\beta_{i}^{\prime}$ from the expression of $B$ for all $i$.

Computing the mean of $g$ and applying the conditions on $n_{i}$ in (5) (which are the same for $w_{i}$ and $w_{i}^{\prime}$ ) give $g(y)=$ $\left[1 / N \sum_{i=1}^{N} w_{i}^{\prime}(y)-A / N \sum_{i=1}^{N} w_{i}(y)\right]=0$ for all $y \in[0,1]$. This means that $w_{i}(y)=w_{i}^{\prime}(y)$ for all $y \in[0,1]$ and $i$.

Finally, if we replace $A, B$, and $g$ by their value, respectively, we obtain $\mu(y)=\mu^{\prime}(y)$ for all $y \in[0,1]$ which completes the proof. In fact, the CISA model is identifiable.

\section{ACKNOWLEDGMENT}

This work was supported by a doctoral grant referenced as 394114L from Provence, Alpes, Côte d'Azur (PACA) region, France, and MXM Laboratories, Vallauris, France.

\section{REFERENCES}

[1] J. O. Ramsay and B. W. Silverman, Functional Data Analysis, Springer Series in Statistics, Springer, New York, NY, USA, 1997.

[2] S. Boudaoud, H. Rix, and O. Meste, "Integral shape averaging and structural average estimation: a comparative study," IEEE Transactions on Signal Processing, vol. 53, no. 10, pp. 36443650, 2005.

[3] S. Boudaoud, H. Rix, and O. Meste, "Providing sample shape statistics with FCA and ISA approaches," in Proceedings of the 13th Workshop on Statistical Signal Processing (SSP '05), pp. 443-448, Bordeaux, France, July 2005.

[4] H. Rix, O. Meste, and W. Muhammad, "Averaging signals with random time shift and time scale fluctuations," Methods of Information in Medicine, vol. 43, no. 1, pp. 13-16, 2004.

[5] H. Rix and J. P. Malengé, "detecting small variations in shape," IEEE Transactions on Systems, Man, and Cybernetics, vol. 10, no. 1, pp. 90-96, 1980.

[6] H. Rix, S. Boudaoud, and O. Meste, "Clustering signal shapes: applications to P-waves in ECG," in Proceedings of the 2nd European Medical and Biological Engineering Conference (EM$B E C^{\prime}$ '02), pp. 364-365, Vienna, Austria, December 2002.

[7] S. Boudaoud, H. Rix, J. J. Blanc, J. C. Cornily, and O. Meste, "Integrated shape averaging of the P-wave applied to AF risk detection," in Proceedings of the 30th Annual International Conference of Computers in Cardiology, vol. 30, pp. 125-128, Thessaloniki, Greece, September 2003.

[8] S. Boudaoud, C. Heneghan, H. Rix, O. Meste, and C. O’Brien, "P-wave shape changes observed in the surface electrocardiogram of subjects with obstructive sleep apnoea," in Proceedings of the 32nd Annual International Conference on Computers in Cardiology, pp. 359-362, Lyon, France, September 2005.

[9] T. Young, L. Evans, L. Finn, and M. Palta, "Estimation of the clinically diagnosed proportion of sleep apnea syndrome in 
middle-aged men and women," Sleep, vol. 20, no. 9, pp. 705706, 1997.

[10] A. S. M. Shamsuzzaman, B. J. Gersh, and V. K. Somers, “Obstructive sleep apnea: implications for cardiac and vascular disease," Journal of the American Medical Association, vol. 290, no. 14, pp. 1906-1914, 2003.

[11] R. S. T. Leung and T. D. Bradley, "Sleep apnea and cardiovascular disease," American Journal of Respiratory and Critical Care Medicine, vol. 164, no. 12, pp. 2147-2165, 2001.

[12] T. Penzel, J. McNames, P. de Chazal, B. Raymond, A. Murray, and G. Moody, "Systematic comparison of different algorithms for apnoea detection based on electrocardiogram recordings," Medical and Biological Engineering and Computing, vol. 40, no. 4, pp. 402-407, 2002.

[13] J. O. Ramsay and X. Li, "Curve registration," Journal of the Royal Statistical Society. Series B, vol. 60, no. 2, pp. 351-363, 1998.

[14] D. Gervini and T. Gasser, "Self-modelling warping functions," Journal of the Royal Statistical Society. Series B, vol. 66, no. 4, pp. 959-971, 2004.

[15] W. H. Lawton, E. A. Sylvestre, and M. S. Maggio, "Self modeling non linear regression," Technometrics, vol. 14, no. 3, pp. 513-532, 1972.

[16] A. K. Jain, R. P. W. Duin, and J. Mao, "Statistical pattern recognition: a review," IEEE Transactions on Pattern Analysis and Machine Intelligence, vol. 22, no. 1, pp. 4-37, 2000.

[17] J. Carlson, R. Johansson, and S. B. Olsson, "Classification of electrocardiographic P-wave morphology," IEEE Transactions on Biomedical Engineering, vol. 48, no. 4, pp. 401-405, 2001.

[18] P. E. Dilaveris and J. E. Gialafos, "Future concepts in Pwave morphological analyses," Cardiac Electrophysiology Review, vol. 6, no. 3, pp. 221-224, 2002.

S. Boudaoud was born in Béjaïa, Algeria, in May 1975. He received the Engineer grade in electrical and electronic engineering from the University A. Mirra of Béjaïa, Algeria, in 1998, the M.S. degree in electrical and electronic engineering from the University F. Abbas of Sétif, Algeria, in 2001, and the M.S. degree in signal processing and communication from the University of Nice-Sophia Antipolis, France, in

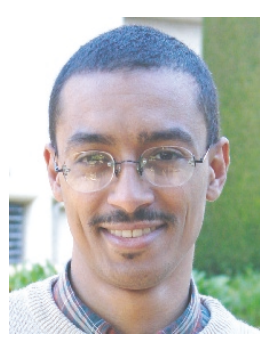
2002. He completed the Ph.D. degree in automatic, signal, and image processing in 2006 at the University of Nice-Sophia Antipolis. Currently, he is a Temporary Assistant Professor at the BIOMED Group, Laboratory of Informatic, Signals \& Systems of Sophia Antipolis, France. His research interests are in signal processing and modeling applied to biomedical fields.

H. Rix received the M.S. degrees in astrophysics (1968) and applied mathematics (1969), the "Doctorat de Spécialité" degree in astrophysics (1970), and a "Doctorat d'Etat" in sciences (1980) from the University of Nice, France. He has been a Professor with the University of Nice since 1987. His research field is signal processing mainly applied to biomedical signals. At the Laboratory of Informatics, Signals \& Systems of Sophia Antipolis, he heads the BIOMED Group.

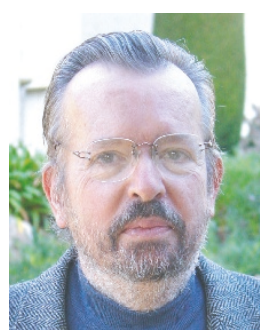

O. Meste received the M.S. degree in automatic and signal processing and the $\mathrm{Ph}$.D. degree in scientific engineering from the University of Nice-Sophia Antipolis, France, in 1989 and 1992, respectively. He is currently working as a Professor at the University of Nice-Sophia Antipolis and as a Researcher at the Biomed Project of the I3S Laboratory. His research interests are in digital processing, time-frequency representations, and modeling to biological signals and systems.

C. Heneghan was born in Dublin, Ireland, in 1968 and received the B.E. degree in electronic engineering from University College Dublin, in 1990 and the Ph.D. degree in electrical engineering from Columbia University, New York, NY, in 1995. He is currently an Associate Professor in the School of Electrical, Electronic, and Mechanical Engineering at University College Dublin, as well as serving as the Chief Scientific Offi-

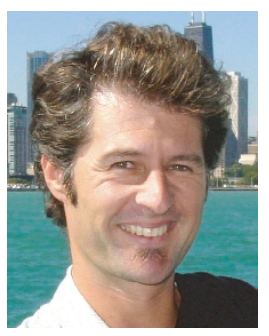
cer of BiancaMed Ltd., an ambient health and wellness monitoring company. He has previously been the Director of Tele-Informatics at the New York Eye and Ear Infirmary, a Visiting Associate Professor at Stanford University's Information Systems Laboratory, and a Visiting Researcher at the Laboratoire Informatique Signaux et Systemes de Sophia Antipolis (I3S). He is a Member of the IEEE Engineering in Medicine and Biology Society, the Signal Processing Society, and the Communications Society. He is a reviewer for several journals including the IEEE Transactions on Biomedical Engineering and IEEE Transactions on Signal Processing. His research interests include signal processing for biomedical applications and signal processing for communications.

C. O'Brien was born in Dublin, Ireland, in 1978 and received the B.E. degree in electronic engineering from University College Dublin in 2000. She completed the Ph.D. degree in electronic engineering in University College Dublin in 2006. Her thesis topic was based on signal processing of a reduced number of biomedical recordings for automated detection of events during sleep such as sleep apnea or sleep staging. The signal

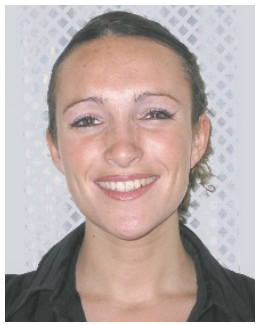
processing involved is based on pattern recognition and signal separation. She is a Member of Engineers Ireland.

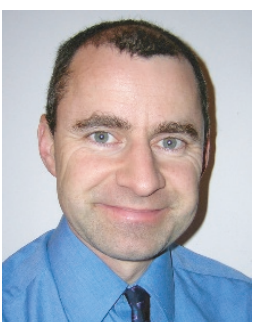

\section{(1)}

